www.jmscr.igmpublication.org Impact Factor 5.244

Index Copernicus Value: 5.88 ISSN (e)-2347-176x ISSN (p) 2455-0450 crossref DOI: http://dx.doi.org/10.18535/jmscr/v4i6.20

\title{
Use of Dexemedetomodine or Buprenorphine Along with Bupivacaine for Ultrasound Guided Brachial Plexus Block: A Comparative Randomized Prospective Study (Original Research Article)
}

\author{
Authors \\ Dr S.Sivakumar M.B.B.S.,M.D ${ }^{1}$, Dr K.Rajarajeswaran M.B.B.S.,M.D² \\ ${ }^{1}$ Assistant Professor, Dept of Anesthesia and Critical Care, Madha Medical College Hospital and Research \\ Institute, Chennai \\ Email:drshiva187@gmail.com \\ ${ }^{2}$ Assistant Professor, Dept of Anesthesia and Critical Care, Madha Medical College Hospital and Research \\ Institute, Chennai \\ Email: rajkv9001@gmail.com \\ Corresponding Author \\ Dr S.SIVAKUMAR, M.B.B.S.,MD
}

\begin{abstract}
Aim and background: Brachial plexus block is one of the commonest regional anesthesia technique used for Upper limb surgeries in orthopedic and plastic reconstructive cases. With the use of ultrasound the accuracy and success rate of the nerve block is improved. Adding Adjuvant like opioids and $\alpha-2$ adrenergic agonist to local anesthetic agent have increased the duration of analgesia. Aim of our study is to compare the effect of dexmedetomidine and buprenorphine added to local anaesthetics in ultrasound guided brachial plexus block in terms of onset and duration time of the sensory and motor block, their side effects and complication.
\end{abstract}

Methods: 60 ASA I and II patients posted for upper limb surgeries were randomly divided into Group I : Dexmedetomidine $1 \mathrm{mcg} / \mathrm{kg}$ added to bupivacaine $2 \mathrm{mg} / \mathrm{kg}$ and Group II : Buprenorphine $0.3 \mathrm{mg}$ added to to bupivacaine $2 \mathrm{mg} / \mathrm{kg}$. Vitals signs, motor blockade and sensory blockade onset and duration were recorded peri-operatively.

Results: Onset of sensory and motor block are similar in both group. The duration of sensory and motor blockade are longer in group II than in group I. Motor blockade mean duration in group I and II are $660.34 \pm 40.6$ and $740.18 \pm 28.4$ respectively. Postoperative pain and PONV are reduced in both groups. No procedure related complication or drug related side effects were recorded in our study.

Conclusion: Use of ultrasound for brachial plexus block improves the quality of the blockade with minimal drug volume and therefore reducing their systemic toxicity. Both dexmedetomidine and buprenorphine added to bupivacaine increases duration of sensory and motor blockade about 2 -3 times that of brachial plexus block with local anesthetic alone and with reduced postoperative pain and PONV.

Keywords: 1.Brachial plexus block 2. Ultrasound 3.dexmedetomidine 4.Buprenorphine.5.Motor blockade 6. visual analogue score (VAS). 


\section{INTRODUCTION}

Brachial plexus block is common peripheral nerve block in orthopedic and plastic forearm and arm surgeries. Peripheral nerve block can be performed by elicitation of parasthesia blindly, with electrical nerve stimulator or with the guidance of ultrasound. Due to development in high frequency ultrasound imaging, its usage in regional anesthesia had increased recently. Ultrasound guided peripheral nerve block is a recent technique for accurate and improved quality nerve block therefore reducing block failures and to avoid procedure related complications like intraneural, intrathecal and intravascular injections. Many drugs such as Clonidine, Fentanyl, buprenorphine, ketamine, Midazolam, dexmedetomidine, morphine have been used as adjuvants to local anesthetic agents to quicken the onset and prolong the block duration with better post-operative pain relief. This technique can be useful in ambulatory day care surgeries, and for better pain relief in patient with co-morbid condition for whom intravenous NSAID and opioids are not advisable (e.g bronchial asthma ,chronic renal failure, COPD). Since these adjuvants have receptor mediated analgesic effects at the spinal and supra-spinal level and these finding has motivated research on peripheral analgesic action of these drugs and their benefit of reduced systemic side effect while giving in nerve blocks. Since peripheral nerves have opioid receptors, many studies have been conducted to understand the effect of narcotics added with local anesthetic agent. Some studies have concluded that, buprenorphine an agonistantagonist opioid added to bupivacaine, provided a longer period of postoperative analgesia than other opioid variants. Similar results were reported, when Clonidine, a partial $\alpha-2$ adrenoceptor agonist used along with local anesthetic agents also prolongs the duration of analgesia. The $\alpha 2: \alpha 1$ selectivity of dexmedetomidine is 8:1 as that of Clonidine and this property of high specificity for $\alpha 2$ subtype makes it better sedative analgesic without hemodynamic changes like bradycardia or hypotension as compared with clonidine. Dexmedetomidine has frequently being used in intensive care unit as sedation for intubated patients on mechanical ventilator, fibreoptic bronchoscopy guided awake intubation, as adjuvant in spinal and epidural anesthesia. But dexmedetomidine usage in peripheral nerve block study reports are limited.

This study compares the effect of dexmedetomidine and buprenorphine added to bupivacaine in ultrasound guided brachial plexus block in terms of onset and duration time of the sensory and motor blocks, their side effects and complication.

\section{METHODS AND MATERIALS}

Study design: Randomized prospective control double blinded study done after institutional ethical committee approval and informed written consent from the patients.

60 patients admitted for upper limb surgery were randomly allotted. (30 in each group)

Group I : Dexmedetomidine $1 \mathrm{mcg} / \mathrm{kg}$ added to bupivacaine $2 \mathrm{mg} / \mathrm{kg}$ (total - $20 \mathrm{ml}$ solution)

Group II : Buprenorphine $0.3 \mathrm{mg}$ added to to bupivacaine $2 \mathrm{mg} / \mathrm{kg}$ (total - $20 \mathrm{ml}$ solution)

\section{Inclusion criteria:}

Physical status-ASA 1 and 2, Age- $20-70$ years old, Upper limb surgeries.

\section{Exclusion criteria:}

Physical status - ASA 3,4, and 5, Age - less than $20 \mathrm{yr}$ and more than $70 \mathrm{yrs}$, Co-morbid condition - CAD, CRF, COPD patients, Psychiatric illness and neuromuscular disorder.

Preoperatively intravenous fluid started. Standard monitors (E.C.G,SPO2,NIBP,H.R,) attached to the patient and oxygen $5-6 \mathrm{~L} / \mathrm{min}$ given via face mask. With ultrasound guidance depending upon the surgery, one of the brachial plexus block approach performed (eg. Arm surgery interscalene approach, forearm surgey supraclavicular approach, hand surgery infraclavicular or axillary approach) and total volume of $20 \mathrm{ml}$ drug solution injected. After the 


\section{JMSCR Vol||04||Issue||06||Page 10802-10806||June}

procedure the following parameters were assessed intra-operatively : 1 .Onset of sensory blockade by pinprick test using $27 \mathrm{G}$ needle, 2. Onset of motor blockade - by grading motor function at shoulder joint (interscalene approach), at elbow joint (supraclavicular approach), at wrist joint and finger movements (axillary and infraclavicular approach)[table 1]. Vital signs were monitored throughout the surgery and during PACU stay. Visual analogue scoring scale used for assessing postoperative pain (VAS 0 - 10 score. 0 - nil pain , 10 - worst pain).statistical analysis done using unpaired $\mathrm{T}$ test. $(\mathrm{P}$ value $>0.05$ insignificant, $\mathrm{P}<$ 0.05 significant difference, $\mathrm{P}<0.01$ highly significant difference).

\section{RESULTS}

Demographic data of the study group are given in table 2. The onset of sensory and motor blockade in group and group II are similar and difference between them is statistically insignificant $\mathrm{P}>0.05$. The duration of sensory blockade in group I and group II are $720.24 \pm 80.3$ and $880.64 \pm 42.4$ respectively. The duration of motor blockade in group I and group II are $660.34 \pm 40.6$ and 740.18 \pm 28.4 respectively [ Table 3]. There is significant difference between the groups in terms of sensory and motor block duration $\mathrm{P}<0.05$. There was no major adverse event during the procedure or during intraoperative period. The vital parameters are within safer limit during intraoperative period in both groups. All the patient were shifted to PACU for observation. Vitals signs and any complication were recorded in PACU. Postoperative pain were assessed using VAS scoring scale. The mean duration of analgesia in group I and group II are 840.36 \pm 20.68 and $960.48 \pm 32.56$ respectively[Table 4]. When pain score is > 4 fentanyl $2 \mathrm{mcg} / \mathrm{kg}$ given with or without NSAID. The VAS score at 12, 24, 36, 48 hour were similar in both groups. Vitals were stable throughout the postoperative period and there was no significant systemic toxicity due to drug or procedure related complication noted. PONV were noted in 2 patient in group 1 and 6 patients in group II and it was treated with intravenous ondensetron.

Table 1 .Assessment Scale for Sensory And Motor Blockade.

\begin{tabular}{|l|l|l|}
\hline \multicolumn{1}{|c|}{ scale } & Sensory blockade & Motor blockade \\
\hline No block & Normal sensation & Full muscular action \\
\hline partial block & Decreased sensation & Decreased muscular action \\
\hline Complete block & Total loss of sensation & Complete loss of action \\
\hline
\end{tabular}

Table 2 .Demographic data expressed as mean \pm SD

\begin{tabular}{|l|l|l|}
\hline variables & Group I & Group II \\
\hline Age & $48.2 \pm 18.6$ & $45.7 \pm 16.3$ \\
\hline sex & $18 / 12$ & $20 / 10$ \\
\hline Height & $169.4 \pm 10.2$ & $165.5 \pm 12.1$ \\
\hline weight & $62.2 \pm 11.2$ & $60.8 \pm 12.3$ \\
\hline Duration of surgery & $140.46 \pm 34.3$ & $138.32 \pm 28.3$ \\
\hline
\end{tabular}

Table 3. Intra-operative outcomes in minutes

\begin{tabular}{|l|l|l|}
\hline Variables & Group I & Group II \\
\hline Onset of sensory blockade & $5.8 \pm 1.4$ & $6.1 \pm 2.2$ \\
\hline Onset of motor blockade & $6.6 \pm 4.2$ & $7.0 \pm 1.8$ \\
\hline Duration of sensory blockade & $720.24 \pm 80.3$ & $880.64 \pm 42.4$ \\
\hline Duration of motor blockade & $660.34 \pm 40.6$ & $790.18 \pm 28.4$ \\
\hline
\end{tabular}


Table 4 post-operative outcome

\begin{tabular}{|l|l|l|}
\hline Variables & Group I & Group II \\
\hline Duration of analgesia (min) & $840.36 \pm 20.68$ & $960.48 \pm 32.56$ \\
\hline VAS score at 12 hour & $1.8 \pm 0.8$ & $1.4 \pm 0.4$ \\
\hline VAS score at 24 hour & $5.4 \pm 1.2$ & $6.2 \pm 0.8$ \\
\hline VAS score at 36 hour & $6.3 \pm 0.4$ & $6.4 \pm 0.6$ \\
\hline VAS score at 48 hour & $6.2 \pm 0.6$ & $6.2 \pm 0.4$ \\
\hline
\end{tabular}

\section{DISCUSSION}

Brachial plexus nerve block is an better alternative to general anesthesia especially in patients with co-morbid condition like CAD,COPD,CRF for whom general anesthesia possess high risk to their life and for whom better postoperative analgesia will improve faster and safer recovery without adverse events . Other advantage of brachial plexus block are better intra operative and postoperative analgesia and such presumptive analgesia will reduce the perioperative analgesic doses (opioids and NSAID ) which would be beneficial to patients with medical conditions like COPD, bronchial asthma, $\mathrm{CRF}^{[1,2]}$. Use of ultrasound for brachial plexus block improves the quality of the block and reduces the failure rate when compared with parasthesia technique and electrical nerve stimulator technique. The duration of analgesia in brachial plexus block with local anesthetics alone will be $6-8$ hours ${ }^{[2]}$. This study was done to analyze whether addition of adjuvant to local anesthetics will prolong the duration of analgesia, motor and sensory blockade if so, these finding could be used to improve better patient recovery with comfortable hospital experience. While performing block under ultrasound guidance, a volume of $20 \mathrm{ml}$ local anesthetic with adjuvants was adequate for complete block in all patients as reported in other studies ${ }^{[3,4]}$. These doses of buprenorphine $(0.3 \mathrm{mg})$ and dexmedetomidine $(1 \mathrm{mcg} / \mathrm{kg})$ were selected from earlier studies ${ }^{[6,7,8]}$. The onset of sensory and motor blockade in group I and II are same but faster than in brachial plexus block with local anesthetic agent alone ${ }^{[6]}$. The duration of sensory blockade and motor blockade in group II are longer than group II. But adding these adjuvants, duration of motor and sensory blockade is increased $2-3$ times that of local anesthetic alone ${ }^{[6,7]}$. Post operative pain are better controlled with minimal doses of fentanyl and NSAIDS with reduced PONV incidence. These findings of our study is comparable with other studies which were done with similar objective ${ }^{[7,8,9]}$. Use of these adjuvants in peripheral nerve block reduces its systemic side effects like respiratory depression, hemodynamic changes, nausea and vomiting which were reflected in our study that none of the patient in our study have major side effects or complication during the study period ${ }^{[9,10]}$.

\section{CONCLUSION}

In conclusion, use of ultrasound for brachial plexus block improves the quality of the blockade with minimal drug volume, fastens the onset of the blockade and minimizes the procedure related complication. Both dexmedetomidine and buprenorphine added to local anesthetic increases duration of sensory and motor blockade about 2 3 times that of brachial plexus block with local anesthetic alone and with reduced postoperative pain and PONV. In view of cost-effectiveness, easy availability, and longer duration of analgesia, this study suggest, buprenorphine $(0.3 \mathrm{mg})$ is better choice than dexmedetomidine as an adjuvant in brachial plexus block.

\section{REFERENCES}

1. Winniee A.P, Collinn V.J. subclavian perivascular technique of brachial plexus block anesthesia. Anaesthesiology 1964; 25: 353.

2. Fischer HJ. Brachial plexus block. In : principle and practices of regional anesthesia; Wildsmit JW, Armitagee E.N, 
Mac Clure J.H, editors, 3rd edition. London: Churchil Livingstonee,2003: 194203.

3. Sites B.D, Tanzer A.H, Herick M.D, Gillon C, Antonakaakis J, Richinns J, Beach ML. Incidence of postoperative neurologic symptom associated with 12,666 ultrasound-guided nerve blockReg Anesth Pain Med 2012;37:478-483

4. Klastad , Sauter A.R, Doddgson MS. Brachial plexus nerve block with or without ultrasound guidance. Cuurr Opinn Anesthesiol 2009; 22 (5):655-665

5. Esmoglu A, Mizrak AA, Akkin A, Booyaci A. Addition of Dexmeditomidine to lignocaine intravenous regional anesthesia

6. Fukusina K, Nishimhi Y, Mori K. Effect of epidural-dexmedetomidine on sympathetic activity and postop pain in man. AnesthAnal 1996; 82: S111.

7. Damin B.M, Colinn J. L., MacCartney, and Vincentw. S., Novel Analgesic Adjuncts used for Brachial Plexus Block: A Systematic Review. A\&A 2000; 90: 5, 1123-1128.

8. Dobbkin AB. Esposit B, Phillbin C . Double blinded evaluation of buprenophine for post op pain. Canadian Anaes Soc. 1977; 24; 194-201.

9. Cahil J, MurphyD, et al. Epidural buprenorphne for pain relief in major abdominalsurgery. a controlledcomparison study.

10. SwamiiSS, KeniyaVM, Ladii SD, RaoR. Comparison ofdexmedetomidine and clonidine (a2 agonist drugs) as anadjuvant to local anesthesia for supraclvicular brachialplexus block: A randomized double-blind prospectivestudy. Indian Jour Anesth 2012;56:243-9. 